\title{
ShopWithMe!: Collaborative Information Searching and Shopping for Online Retail
}

\author{
Yanjun Gao \\ The Pennsylvania State \\ University \\ gaoserenayj@gmail.com
}

\author{
Madhu Reddy \\ Northwestern University \\ mreddy@northwestern.edu
}

\author{
Bernard J. Jansen \\ Qatar Computing Research \\ Institute, HBKU \\ jjansen@acm.org
}

\begin{abstract}
We present research on the development and evaluation of a collaborative search and shopping system for online retail tasks based on domain specific product requirements. We describe the design rationale for the system development and inclusion of collaborative features, including search, chat, clip-board, product suggestions, shared views, and shopping cart with a focus on how these features are used for collaborative online retail shopping and information searching and sharing. Our research goal is to understand whether collaborative search tools are useful in supporting actual collaborative online retail shopping tasks for experience goods. We describe system development and report findings from preliminary user studies of the system, using mixed methods analysis, with an emphasis on the qualitative findings. The findings highlight that systems for the online shopping domain can support searching, shared views, and group communication to aid in collaborative shopping for experience goods by improving information sharing among group members. Implications are that ecommerce systems, websites, and web apps should support collaboration based on product types.
\end{abstract}

\section{Introduction}

Information searching can be a social and collaborative activity $[1,2]$ in certain contextual situations. Individuals routinely seek assistance from others, especially online, for help in tasks in which they are uncomfortable or need information [3. 4]. However, most search tools, techniques, and paradigms are designed for single users in a noncollaborative mode. Although there are an increasing number of collaborative search models [5] and systems [6, 7], few of the resulting collaborative systems have gained wide adoption.

One potential reason for this lack of adoption is that collaborative search has primarily been visualized from a general perspective, resulting in collaborative search systems developed for generic search but not for domain specific collaborative search tasks with relatively welldefined goals and objectives [8]. Furthermore, there are many domain dependences, including language, cost, objective, and time that have to be taken into account for developing domain-specific collaborative search tools.

There has been limited research in understanding the collaborative nature and elements of the underlying domain specific tasks, which may have more specific jargon and narrower goals relative to general information tasks. Furthermore, there has been little work in developing domain-specific collaborative search and task-focused systems, as opposed to general web search engines such as Google and Baidu that contain collaborative features [9, 10]. The development of such collaborative domain specific searching systems could improve the effectiveness and efficiency of ecommerce shopping. This is the motivational foundation for the research presented here.

In our present study, we develop and evaluate a collaborative web searching and shopping system for the ecommerce domain, specifically for a collaborative online retail shopping task, where a group of shoppers work together to find a set of needed products [2]. Online shopping has unique domain attributes, such as personal taste and price aspects, among other features [11], and because of this, online shopping requires specialized support [12].

The collaborative system features are based on online retail shopping task attributes, specifically the product type and the unique elements of experience goods, which are those products that must be used before their value can be determined. As such, the research presented here is unique because it is domain-specific and task-focused rather than focused on general collaborative search. We also conducted preliminary user studies of the collaborative shopping system to start to investigate how shoppers interact with the system. The findings from our user studies highlight how collaborative search systems can assist in certain shopping situations, specifically online retail shopping where the shopper desires the input concerning products that requires the expertise of others.

\section{Research Objectives}

We believe that identifying and developing systems that support domain-specific collaborative tasks, in conjunction 
with search, is the key to wider adoption of collaborative searching tools. Although there has been substantial research effort in defining collaborative search [5] and in developing a variety of collaborative search systems [7, 9, $10]$, most of these models and systems have taken a generic view of collaborative searching, ignoring potentially important domain dependent characteristics that may be critical to the design and adoption of collaborative search systems. Therefore, we believe that developing systems for specific domains, where the system attributes reflect and support the underlying collaborative task being addressed, is beneficial for the future direction of collaborative searching, and shopping, systems.

Therefore, our research objective is to investigate how ShopWithMe! Supports collaboration among shoppers during an online retail shopping scenario.

To accomplish this objective, we developed a collaborative search and shopping system, ShopWithMe!, that allows multiple individuals to collaborate together (either synchronously or asynchronously) [4, 5] to accomplish an online retail shopping task. Although possessing some similar collaborative features as other systems [7, 9, 10], our work is anchored in the retail shopping domain, with a specific focus on the unique aspects of experience products [13]. Therefore, ShopWithMe! not only supports collaborative search but more specifically collaborative shopping for specific product types. Therefore, it is task- and domain- specific. We followed the system development with preliminary user studies to examine how shoppers interact with the system

\section{System Development}

Many tasks have unique characteristics that differentiate them from similar tasks in other domains [12, 14]. The central differentiating attribute of online retail shopping is the nature of the product, which relates directly to the shopping task. For online retail shopping, the nature of the product impacts the context of the collaboration around the task and also the collaborating aspects of information searching that aids in successful task competition.

In online retail, consumer products can be classified into three categories based on the nature of the information concerning those products: search products, experience products, and credence products [13].

- Search products are goods where the consumer can easily evaluate the characteristics prior to purchase. An example is a specific book by a given author.

- Experience products are goods where the consumer cannot easily determine the products' characteristics prior to purchase but can determine these characteristics upon purchase, consumption, or use of the goods. An example is a new type of food.

- Credence products are goods where the consumer cannot easily determine the products' quality even after purchase, consumption, or use of the goods. An example is healthcare.

For this project, we focused on experience products, because these goods are naturally the focus of collaborative search and collaborative retail shopping tasks, where information sharing occurs during the shopping activity [15]. Although there are other possible contexts for experience goods, such as online reviews or social media platforms, it also seems natural for collaborative searching where individual can share their opinions and insights.

Search products (e.g., printer cartridges) would not generally be considered a focus for collaborative shopping because the price and quality can easily be determined by the individual via straightforward information searching and retrieval. Credence products (e.g., health care) often require expert or third-party expertise, so collaborating with non-expert peers might not be beneficial.

However, experience products fit nicely with the concept of collaborative search in an online retail environment because the advice and assistance of others can reduce the uncertainty concerning the product by viewing search for these goods within a collaborative context as similar to a learning endeavor $[16,17]$.

We also hypothesize that experience goods can be situational or contextual dependent. For example, a piece of clothing can be inherently an experience product and additionally the appropriateness of the clothing can be tied to an event, reinforcing the collaborative search and nature of the online shopping task.

This view of the online retail shopping information needs based on product types is supported by prior work [18]. Researchers have highlighted that collaborative search tools typically offer two types of affordances that separate them from individual search systems [9, 19]:

(a) awareness features: sharing of queries, and comments among team members, and

(b) division of labor features: ability to manually divide the tasks, both information and underlying

Based on this concept of experience goods and collaborative affordances of awareness and division of labor, we developed ShopWithMe! specifically to support online retailing shopping. ShopWithMe! aids in the collaborative information search needed to complete or improve the effectiveness of the task, both synchronously and non- synchronously [20]. We employed a featuredriven development (FDD) software development approach. FDD is an iterative and incremental software development process that focuses on crafting of the overall model, list of features, feature planning, feature design, and feature building.

ShopWithMe! allows multiple users to conduct web search and product retrieval independently [19], while also seamlessly interacting with others collaboratively to accomplish the retail shopping task. 


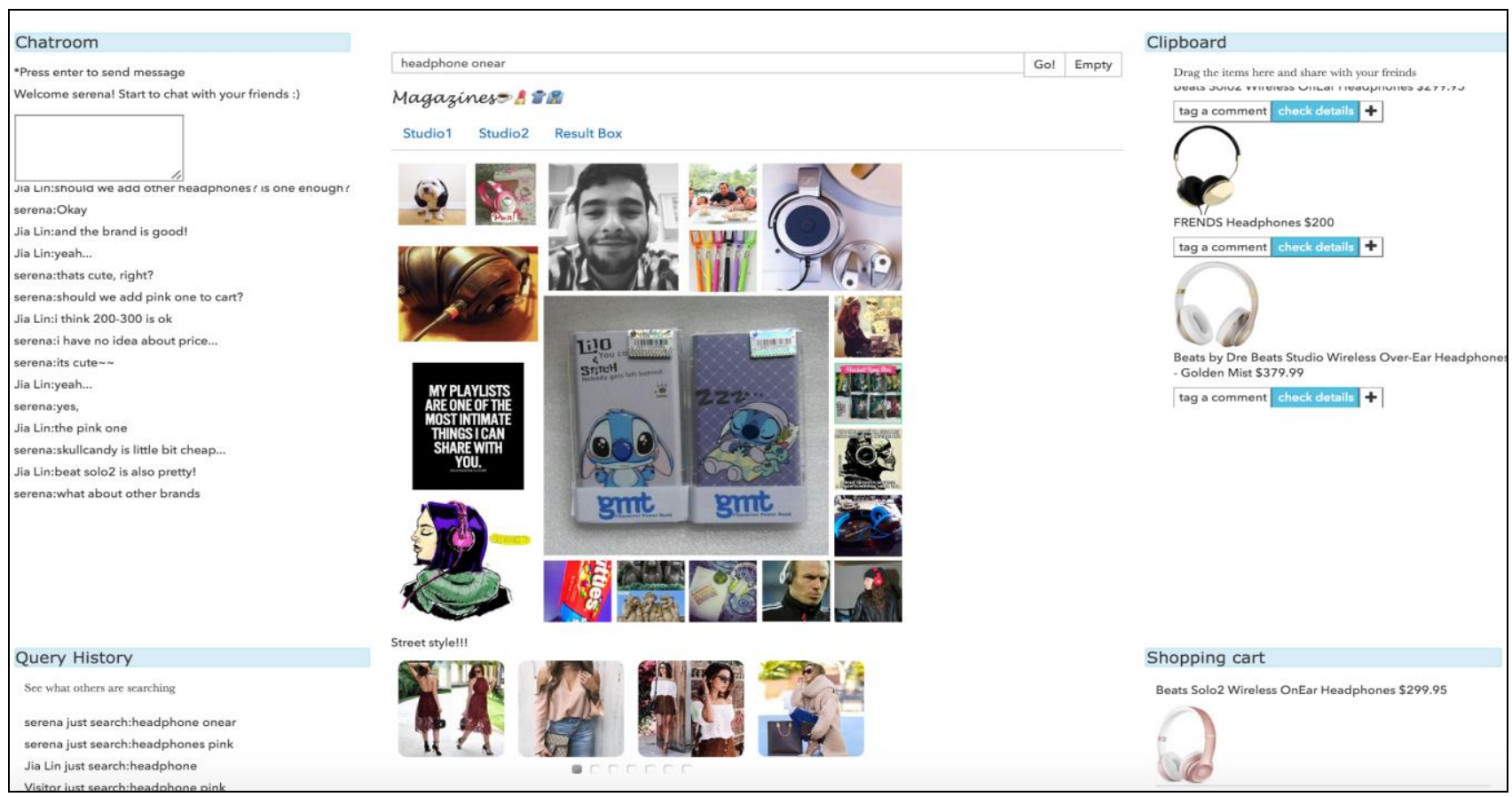

Figure 1. Screenshot of user interface showing collaborative components of search section, chatroom, clipboard, query history, shopping cart.

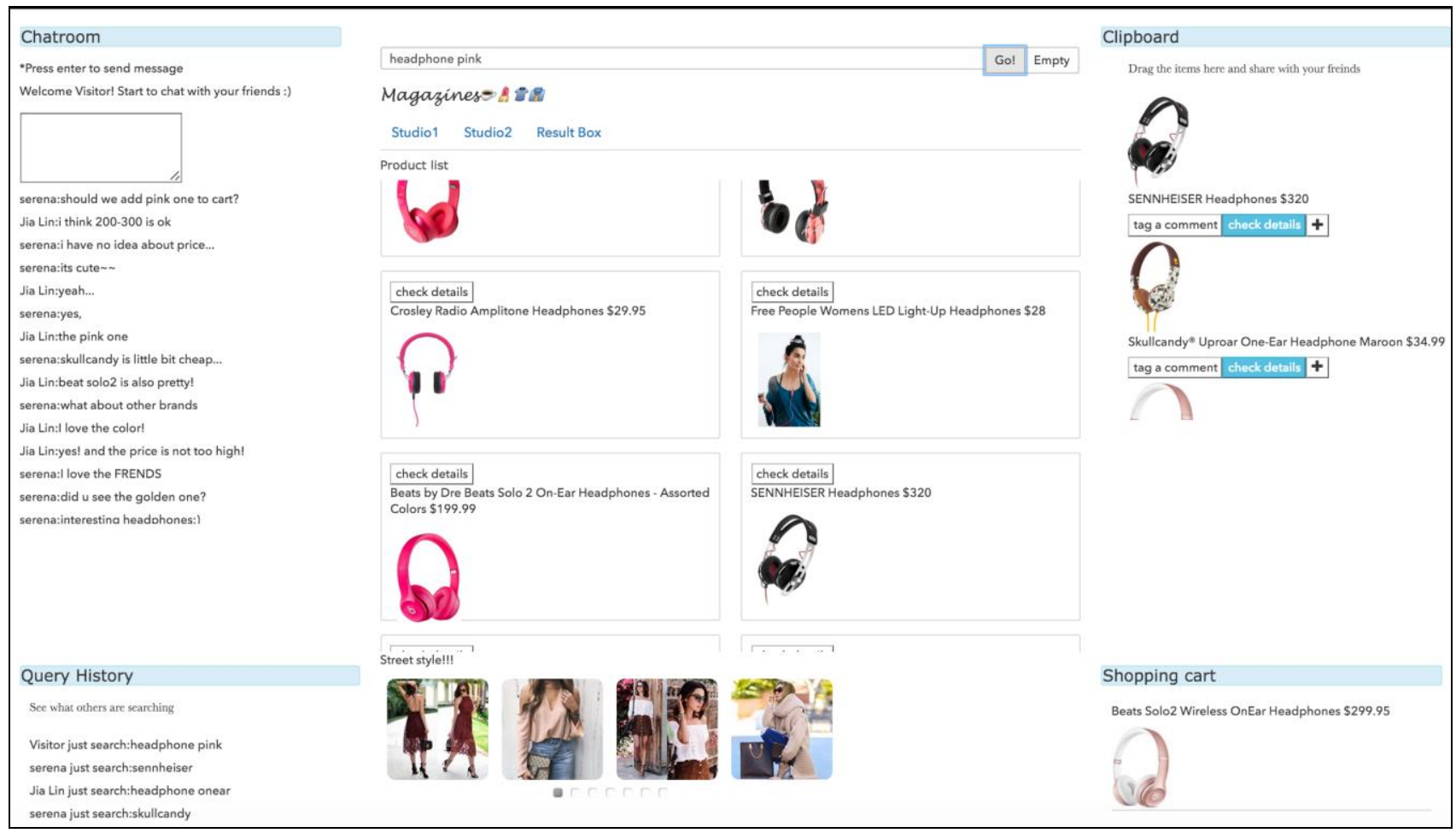

Figure 2. Screenshot of Magazine Portion of Interface Inspiration for Her via Instagram Account 'headphone pink'. 


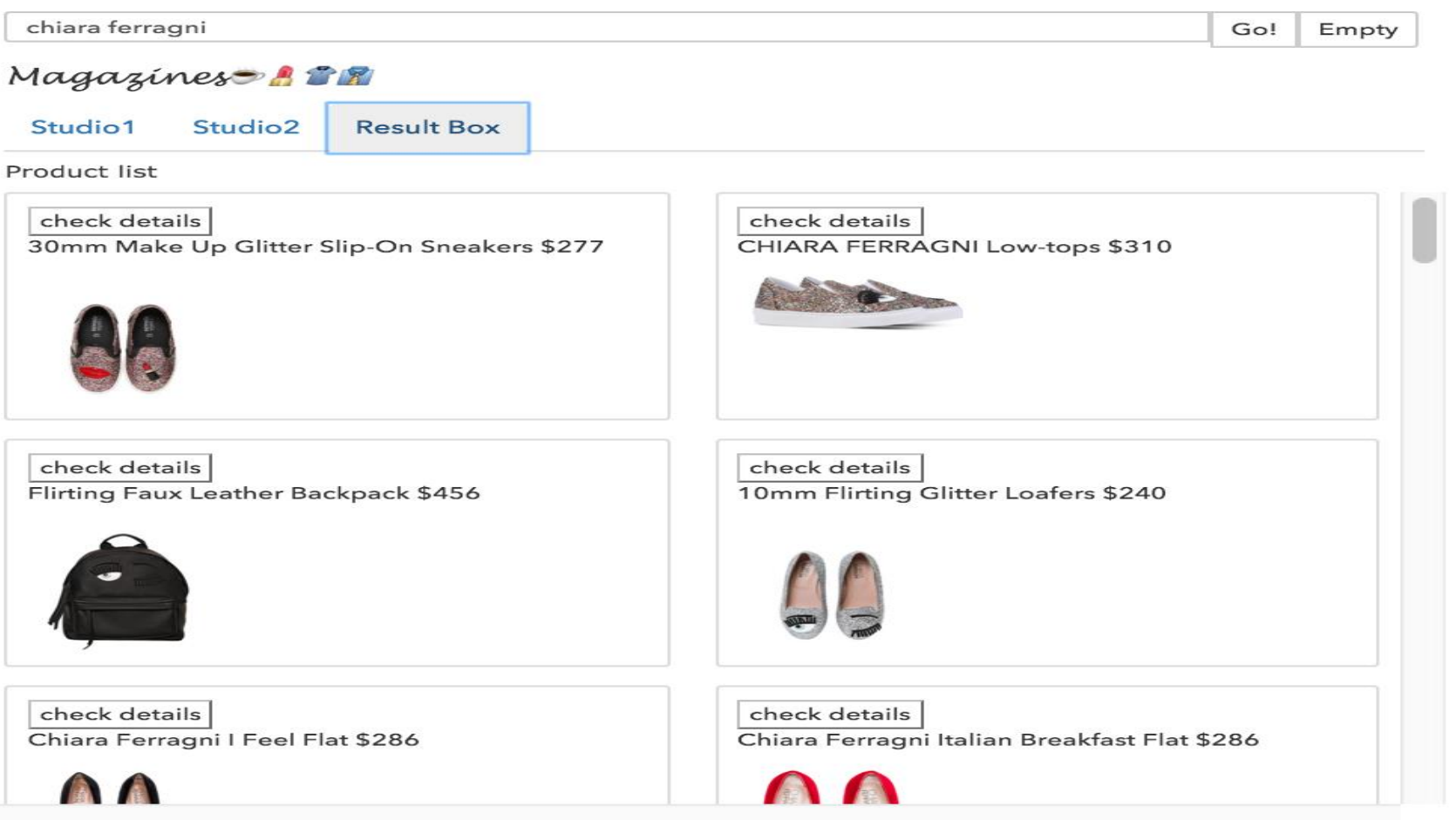

Figure 3. Search Results with Search Terms 'chiara ferragni'.

The system front-end is built on HTML, CSS, and JavaScript, all well-established web scripting languages. The back-end is Django (an open source web framework) and SQLite (a C-based SQL database engine). We run Twisted (event-driven networking engine) as the web server, which supports all synchronous features. The retail product database is real-world (i.e., contains real products) and is updated in real time during the online shopping sessions. The product database allows access to individual products and meta-data in real time via an API to the ShopStyle shopping site, (http://www.shopstyle.com/), a popular retailer product website. So, in addition to our conceptual framework of collaborative information search within the online retail domain, requiring recognition of product types, awareness features, division of labor, and communication among group members for the virtual workspace [21], we have also developed a robust prototype of a collaborative search and shopping system.

In the following sections, we first describe the development of the system and its individual components, focusing on the user interface and the collaborative aspects, which are most relevant to the collaborative aspects of information searching in the online retail domain with experience goods.

\section{User Interface}

The user interface (Figure 1) has two sections, one focuses on the individual features, and the other focuses on the collaboration aspects [5].

The individual section is composed of a magazine' section (Figure 2) [22] and search box (Figure 3). The collaboration section includes a query history (Figure 4), chatroom (Figure 5), clipboard (Figure 6), and shopping cart (Figure 7) [21].

\subsection{Individual Section of User Interface}

This section is composed of the two following features:

Search Box: The search box is where the user can type terms to query for products. Each item in the list of matched results contains the name, brand, price, and picture of the product. A 'check details' button forwards the user to a complete item description page (Figure 3). Each item in the results list has 'draggable' and 'droppable' attribute so that the user can place the item to clipboard and share to other users while they are browsing. The empty button clears the search box and product listing for restarting a search.

Magazine: The magazine is an embedded gallery that allows users to browse and look for product inspirations. Experience goods possess attributes that are not easily searchable by text, so we believe that images of products may be beneficial to the retail 
shopping task. When the user browses the magazine, he can change pages or click on images to see the product details, including item names, brands, and description (see Figure 1).

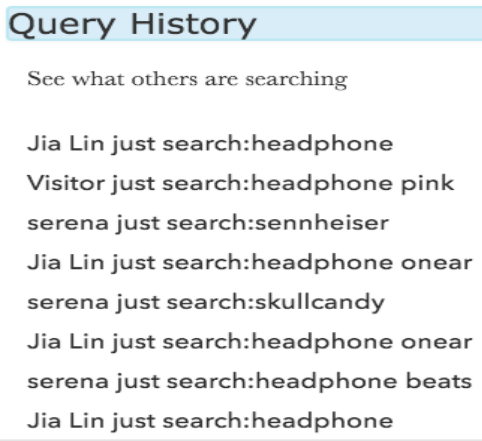

Figure 4. Query History with Individual Queries from the Collaboration Members.

The magazine tab is based on a popular Instagram account targeted at young shoppers. The embedded gallery is implemented via Instush (http://www.instush.com/), which allows for the imbedding of Instagram galleries within applications. These accounts are updated in real time.

So, ShopWithMe! supports individual web searching and web browsing in the ecommerce domain with the search and magazine components, respectively, while also directly support the retail shopping task.

\subsection{Collaboration Section of User Interface}

For collaboration, ShopWithMe! provides an awareness component with the query history attribute, and the chatroom feature allows for division of labor and also discussion and information sharing. At present, the system is designed to support all shoppers online at the same time; however, it could be modified to support shoppers engaging at different times. There are several features in the collaboration section.

Query History: Query history (see Figure 4) is the interface component showing what other members collaborating on the shopping task are searching for. This feature allows searchers awareness of the particular direction that others involved in the shopping task are taking. It can also be a source of additional query terms and provide early insights into product perceptions of the individual shoppers.

Chatroom: This is the main communication tool of the system (Figure 5) for collaboration members. It contains the input box for new posts and also a message archive for historical references. The chatroom has a rollover attribute so that users can keep track of chatting history while searching for information, supporting awareness and division of labor.

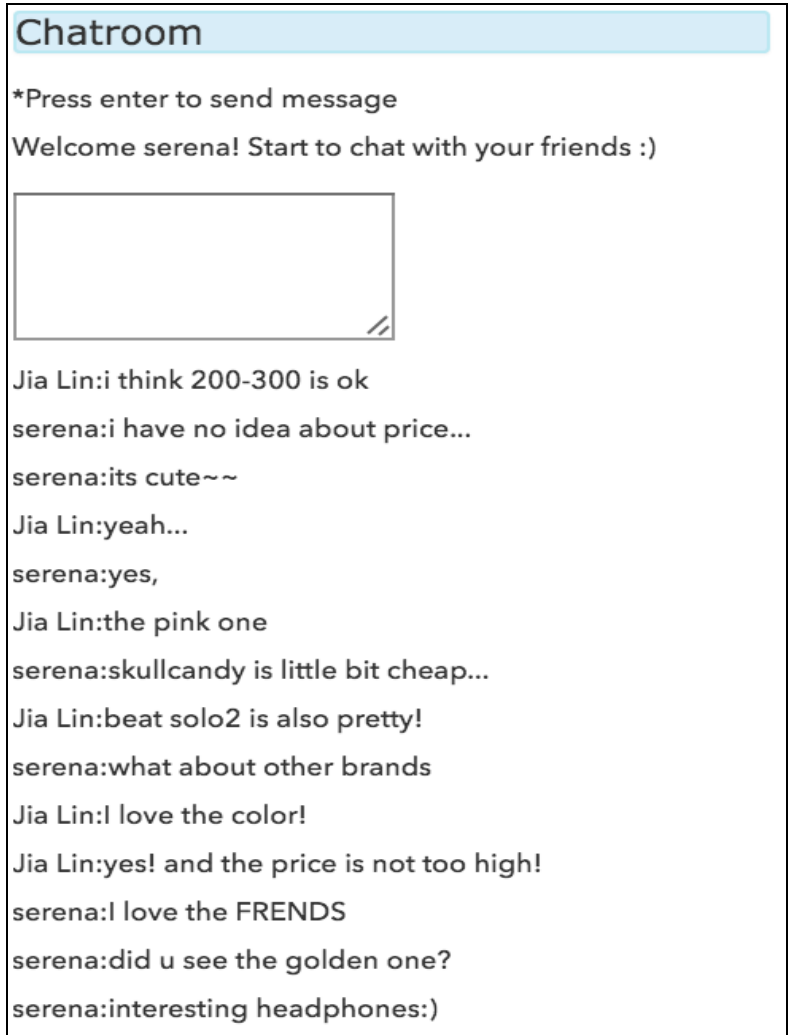

Figure 5. Chatroom with Chat Conversation among Collaboration Members.

The chatroom also allows for feedback from individual members, which we consider essential for shopping for an experience product. This feedback is important because these individuals may have experience with the product themselves that they want to share or may provide perceptions and opinions concerning the product.

Clipboard: The clipboard is the principal image communication feature, allowing individual members to share products that they have found with other members. The clipboard offers cut-and-paste capabilities and sharing of images during shopping sessions (see Figure 6). The product images that individual members paste to the clipboard are immediately available to other members. 


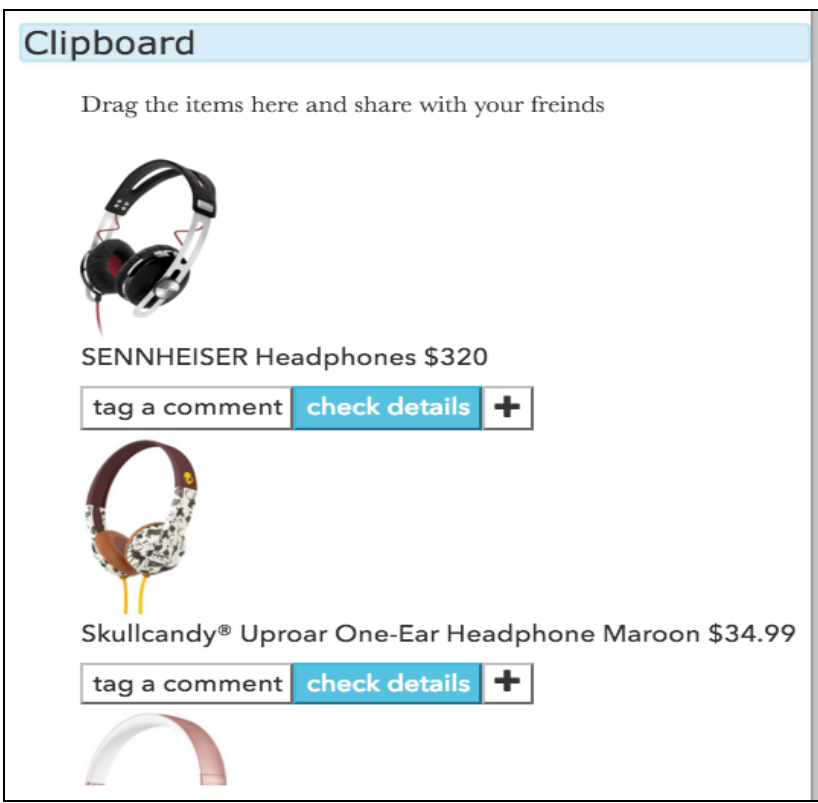

Figure 6. Clipboard with Items from individual Members to Awareness Among Collaborative Members.

Shopping Cart: This is a fully functional shopping cart service, with all members being able to view the items in the shopping cart (as shown in Figure 7).

\section{Shopping cart}

Beats Solo2 Wireless OnEar Headphones \$299.95

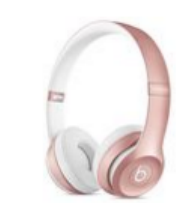

Figure 7. Shopping Cart with Items, Headphones in this Example, viewable to all Members in the Collaboration.

To investigate the effectiveness of ShopWithMe!, we conducted an initial pilot evaluation and user study, with a more complete user study planned for future research.

\section{User Study and Collaborative Investigation}

Currently, we have preliminary results from user studies of different four groups (12 shoppers) engaged in a collaborative shopping task. Of the four groups, there had female participants and one had male participants. We were primarily interested in how the system was used collaboratively by the group members for online retail shopping.

\subsection{Study Design}

We used an online retail shopping domain activity that required collaboration but was also understandable and relatable to the study participants. We did not impose any time constraints on our participants' shopping process. This approach provided ample time for our participants to explore and become familiar with system features, while also allowing us to understand their use of the system during the collaborative shopping activity. The user study framework includes a pre-scenario questionnaire, a brief overview of the collaborative shopping system, the actual user session, and a post scenario interview.

5.1.1. Data Collection. We collected and analyzed the data primarily through qualitative methods. We collected data through directly observing the users interacting with the system and via system logs of search, chat, and clipboard, and interviewing the users about their experience with the system.

5.1.2. Participants. We recruited 12 college-age participants) who were frequent online retailer shoppers and assigned them to 4 groups. The group members knew each other prior to the study [21], which could facilitate the collaborative and advice taking/seeking process.

5.1.3. Shopping Scenario. We designed a search scenario [25] that was complex and nuanced enough to require collaborative search to accomplish the social retail shopping task [25]. Our scenario design was based on two pilot user groups (note: these pilot groups were not used in the data analysis in this paper), where we observed that, if the shopping task was too straightforward and did not emphasis the experience aspect of the product enough, it resulted in individualized searching behavior rather than collaboration and collaborative sense making [20].

We also determined from those two pilots and from prior work on collaborative search [1, 4] that the collaboration must have a focal point or person to provide structure to the collaboration. In our study, we refer to this person as 'group member A'.

Our retail products for the shopping task were experience products. The shopping scenario employed for the user study was: 
You (group member A) are going to an outdoor party for all undergraduates in your department, as a chance to meet the professors, instructors and new friends. You want have to a splendid new outfit for this party. The party will be in the daytime, and the weather will be sunny and dry, with a temperature of about $29^{\circ} \mathrm{C}\left(84^{\circ} \mathrm{F}\right)$. The invitation letter specifies that you have to wear "business casual". You're not sure what to get; it could be a romper or a dress, and you want the input of your friends (group member $B$ and $C$, neither of who have attended this kind of party before themselves). You want the suit to be classy, to reflect your good taste. You want it to be flattering but also appropriate for your age. You also want it to reflect your own unique personality of being an extrovert. So, you want to impress. You don't know what you want at the moment; you will know it when you see it. While you are shopping for your suit, you also need to purchase accessories such as purse, earrings and shoes that will perfectly match your suit. However, if you find a great scarf or shoes, it might influence what suit you get. So, you began the online shopping trip with an ill-defined goal: something classy, flattering, impressive, and unusual. Other interests, blouse, scarf, and shoes, also would be nice but are not the main goal for the online shopping trip.

\section{Results and Discussion} logs.

We begin with some overall results from the system

As shown in Table 1, although all the major components of the collaborative shopping system were utilized, the chat feature was most extensively employed, again highlighting the nature of the retail product, which required comments, insights, and experiences of the collaborative members during the shopping tasks. The clipboard was also used quite extensively, highlighting the interest in 'showing' the product to the other members, rather than just providing textual comments.

The search query were predominantly products (e.g., shoes men business casual, business casual suits men, high heels) but, also some brands (e.g., Dolce \& Gabbana). The chat message dealt much more with opinions and insights on these products (e.g., Do you like blue suits, Nick?, it looks too mature lol, Don't buy that one!!!!) or task division (e.g., I will start choose watch, take a look at the grey one we have on the clipboard, Let's just try to find you something that matches your choice of shoes).

The Query history section provides a great reference for others when users come up with the terms. Participants are aware of the current progress

\begin{tabular}{|l|c|}
\hline \multicolumn{1}{|c|}{ Clipboard } & \multicolumn{1}{|c|}{$\begin{array}{c}\text { Searching and } \\
\text { Shopping } \\
\text { Behaviors }\end{array}$} \\
\hline Number of Queries & 239 \\
\hline Number of Items in Clipboard & 119 \\
\hline Number of items in Cart & 27 \\
\hline Number of Chat Messages & 302 \\
\hline
\end{tabular}

Table 1. Results of keyterms, items in clipboard

and remain consistency in the task. For example, in Group 3, participant B started searching with keywords "men suits casual". Later, participant C composed "shirt blue men oxford" as a query. After participant A typed "watch men", C began to search "leather watch men". The focal of queries kept in the items that could be described as "business casual". In the interview, participant $\mathrm{C}$ mentioned that the query history section was very useful, especially when she did not know where to start.

In the user study sessions, there were several interesting findings. First, we observed how a group of people would virtually gather, using chat, and start discussing about the task they were facing, while using the system. The scenario served as a trigger that stimulated the interactions in terms of deciding the direction of the search and the division of labor. One member, usually member A, would typically "throw out" ideas about the categories of items they were going to search for, and the other members would help narrow down the categories. Furthermore, the discussions centered on aspects that an individual could not easily find by himself, highlighting the nature of the experience product, as being products that one cannot naturally search for information online without the insights of others.

For example, in the chat logs of Group 3the conversation started as following (Please note, $\mathrm{A}$ is the 'group leader' and $\mathrm{B}$ and $\mathrm{C}$ are group members):

Group 3, Participant B: "A, what kind of shoes do you want to wear in the party?"

Group 3, Participant A: "Expensive shoes will be the best."

Group 3, Participant C: "Well,...so good that you have the money, LOL"

Group 3, Participant B: "We can choose some and let him pick."

This interaction is important because during the shopping session, whenever a group member 
discovered a candidate item and dragged it to the clipboard, this sharing action triggered a discussion in the team. Other members actively shared short comments in the clipboard and turned to the chatroom to give more complete feedback. The reciprocation greatly influenced the search direction, including turning to another items, finding more matching items, and changing to a new style.

Table 2 shows the transition from choices of dress to romper in this particular example. The example highlights the importance of others opinions and insights in relation to the unique nature of experience goods.

From our post-session interviews, there were several comments made concerning the nature of the collaborative search process.

Opposite collaboration styles. Group 2 and 4 generated more keyterms, and put more items to the clipboard and shopping cart compared to Group 1 and 3. In the interview, we found that Group 2 and 4 divided the task into individual searches and then combined the results:

Group 2, Participant B: We have co-ordination, we try own best to find, and put as many things as possible.

Group 4, Participant C: We provided the opinions (items), and A selected from them.

On the other hand, Groups 1 and 3 seemed to be blur the boundaries more between individual and group search. When asking if they have clear division of labor when searching, they answered:

Group 1, Participant B: No. My concern is disagreement by other people. If shopping with other people, I will solicit the opinion from them.

Group 3, Participant C: We don't have to. If B and I both agree, we'll put the items to clipboard or shopping cart.

These different collaboration styles highlight an important point about designing collaborative search tools - these tools have to have the flexibility to support different group interaction styles. Consequently, it is essential that we not only understand the domain that these tools will be used in but also the types of group interactions that the tools will have to support.
Experience Product. It was clear that the focus on an experience product influenced the collaborative search process.

\begin{tabular}{|c|c|}
\hline Clipboard & Chatroom \\
\hline $\begin{array}{l}\text { Zimmermann Ryker } \\
\text { Broderie Dress \$530 } \\
\text { Stone_Cold_Fox } \\
\text { Stone Cold Fox } \\
\text { Aden Romper \$345 } \\
\text { For Love \& Lemons } \\
\text { Luau Mini Dress } \\
\$ 197.40 \\
\text { Zimmermann } \\
\text { Admire Cherry } \\
\text { Romper } \$ 530 \$ 371\end{array}$ & $\begin{array}{l}\text { Group 1, Participant A: } \\
\text { do u decide the dress } \\
\text { Group 1, Participant A: } \\
\text { nude one } \\
\text { Group 1, Participant B: i } \\
\text { like the black romper } \\
\text { Group 1, Participant C: } \\
\text { ok } \\
\text { Group 1, Participant A: } \\
\text { sandals one } \\
\text { Group 1, Participant A: } \\
\text { which dress? }\end{array}$ \\
\hline $\begin{array}{l}\text { Joes'Imagine' } \\
\text { Leather Sandal } \\
\$ 86.96 \\
\text { Chanel 'Classic } 2.55 \text { ' } \\
\text { shoulder bag } \$ 3,550\end{array}$ & $\begin{array}{l}\text { Group 1, Participant B: or } \\
\text { the "Club Monaco Tuper } \\
\text { Romper" } \\
\text { Group 1, Participant C: i } \\
\text { like that one too } \\
\text { Group 1, Participant B: } \\
\text { yea } \\
\text { Group 1, Participant C: } \\
\text { what about that purse } \\
\text { Group 1, Participant C: } \\
\text { and the nude shoes? } \\
\text { Group 1, Participant C: } \\
\text { do you like } \\
\text { Group 1, Participant B: or } \\
\text { this one? } \\
\text { Group 1, Participant A: } \\
\text { yep } \\
\text { Group 1, Participant A: } \\
\text { the channel one } \\
\text { Group 1, Participant B: } \\
\text { ok } \\
\text { Group 1, Participant C: } \\
\text { ok }\end{array}$ \\
\hline
\end{tabular}

Table 2. Record from Clipboard and Chatroom talking about 'romper'

Group 3, Participant A; "At the beginning, the idea was not really clear, just a big picture, ... style, details, not clear, but after seeing the pictures, adapting the magazine, not really using magazine, just take a look at it, it became clear gradually" 
Group 4, Participant B: "I was concerned at first because of the possible disagreement and being afraid that we would not find a unique one. There were a bunch of dresses to choose from."

Awareness. The importance of awareness as a needed affordance for collaborative shopping is very apparent, both in the overall goal of the task and in the details. As noted in reactions to the images in the clipboard, highlight the discussion of the perceptive of individual members concerning the product they were searching for:

Group 1, Participant A; "Yes, communication is great. Flattering dress equals a sexy dress. No misunderstanding."

Group 3, Participant A: "I was concerned about what the items. I just starting browsing the items after other people start to drag the items, as I was looking for accessories."

Visual Clues. As interesting aspect of the domain specific nature of ecommerce collaboration with retail products was the importance of the images, prior research has noted the importance of rich media in online shopping [23].

Group 1, Participant A: "Then, I found out that other people did not like the golden bracelet, so I had to browse another bracelet."

Group 3, Participant A: "I was in charge of shoes, but I picked black dress! Clearly! Classic, can match everything."

Group 4, Participant A: "But the dress is so ugly!!!! Check the details!!! OMG"

The sharing of images triggered a set of reactions that would be difficult to elicit using text. Since, shopping is such a visual activity, the images were an important part of the conversation.

\section{Conclusion}

Our research is based on the premise that collaborative search tools could be more effective in supporting collaboration if they are domain targeted. Leveraging our own and other's research on product information types and affordances of collaborative search tools, we developed a collaborative search system for social e-commerce online retail shopping, ShopWithMe!, We evaluated the collaborative aspects and usage of our system using participants engaged in a collaborative shopping task. These findings confirm our premise of domain specific contexts search requirements and will be used to refine the system. We will also conduct research to investigate which of our findings might be transferable to other domains or collaborative search. Further, we aim to conduct a user study comparing the collaborative shopping system with individual searching and sharing platforms. Future research will also examine temporal aspects of retail shopping [26] and leveraging the system searching interactions for commercial goals [27] and leveraging social media information seeking [28][29].

\section{Acknowledgements}

We thank all the users who participated in this study. This research was supported in part from NSF grant (IIS \#0844947).

\section{References}

[1] Morris, M. R. (2013, 23-27 Feb.). Collaborative search revisited. Paper presented at the 2013 Conference on Computer Supported Cooperative Work (CSCW2013), San Antonio, TX.

[2] Shah, C., and González-Ibáñez, R. (2011, 24-28 July). Evaluating the synergic effect of collaboration in information seeking. Paper presented at the 34th International ACM SIGIR Conference on Research and Development in Information Retrieval (SIGIR2011), Beijing, China.

[3] McNally, K., O'Mahony, M. P., Smyth, B., Coyle, M., and Briggs, P. (2011, 13-16 Feb.). Social and collaborative web search: an evaluation study. Paper presented at the 16th International Conference on Intelligent User Interfaces (IUI2011), Palo Alto, CA.

[4] Reddy, M. C., and Spence, P. R. (2008). Collaborative information seeking: A field study of a multidisciplinary patient care team. Information Processing \& Management, 44(1), 242-255.

[5] Reddy, M., and Jansen, B. J. (2007). A model for understanding collaborative information behavior in context: A study of two healthcare teams Information Processing \& Management, 44(1), 256-273.

[6] Capra, R., Chen, A. T., Hawthorne, K., Arguello, J., Shaw, L., and Marchionini, G. (2012). Design and evaluation of a system to support collaborative search. Paper presented at the American Society for Information Science and Technology Annual Meeting (ASIST2012), Baltimore, Maryland. 
[7] González-Ibáñez, R., and Shah, C. (2011, 9-13 Oct.). Coagmento: A system for supporting collaborative information seeking. Paper presented at the American Society for Information Science and Technology Annual Meeting (ASIST2010), New Orleans, LA.

[8] Bashardoost, B. G., Lyons, K, and Leung, R. (2016) A Goal-Oriented Approach for Designing Collaboration Processes. Paper presented at the 49th Hawaii International Conference on System Sciences (HICSS), 757-766.

[9] Morris, M. R. and Horvitz, E. (2007, 7-10 Oct). SearchTogether: an interface for collaborative web search. Paper presented at the 20th annual ACM Symposium on User Interface Software and Technology, Newport, RI.

[10] Paul, S. A. and Morris, M. R. (2009, 4-9 Apr.). CoSense: enhancing sensemaking for collaborative web search. Paper presented at the SIGCHI Conference on Human Factors in Computing Systems (CHI2009), Boston, MA.

[11] Ingham, J. and Cadieux, J. (2016) From E-Shopping System Quality to the Consumer's Intention to Return: A Meta-Analytic Study of the Mediation of Attitude, Usefulness, Enjoyment, and Trust. Paper presented at the 49th Hawaii International Conference on System Sciences (HICSS), 3556-3564.

[12] Zhu, L., Benbasat, I., and Jiang, Z. (2010). Let's shop online together: An empirical investigation of collaborative online shopping support. Information Systems Research, 21(4), 872-891.

[13] Nelson, P. (1970). Information and Consumer Behavior. Journal of Political Economy 78(2), 311-329.

[14] Gao, Y., Reddy, M., and Jansen, B. J. (2016, 7-12 May). Shop Together, Search Together: Collaborative E-commerce. Paper presented at the Conference on Human Factors in Computing Systems (CHI 2016), San Jose, CA., 2081-2087.

[15] Cheung, C. M. K., Liu, I. L. B., and Lee, M. K. O. (2015). How online social interactions influence customer information contribution behavior in online social shopping communities: A social learning theory perspective. Journal of the Association for Information Science and Technology, 66(12), 2511-2521.

[16] Jansen, B. J., Booth, D., and Smith, B. (2009). Using the taxonomy of cognitive learning to model online searching. Information Processing \& Management, 45(6), 643-663.

[17] Moraveji N., Morris, D., Czerwinski, M., and Riche, N. H. (2011, 7-12 May). ClassSearch: facilitating the development of web search skills through social learning. Paper presented at the SIGCHI Conference on Human Factors in Computing Systems (CHI2011), Vancouver, Canada.
[18] Hyseni, H., Brown, C., and\& Gannon, M. (2015, 9-10 July). Shopping and Socialising: Online Journey to Product Evaluation and Purchase in Fashion Retail. Paper presented at the 2nd European Conference on Social Media 2015 (ECSM 2015), Porto Portugal.

[19] Capra, R., Arguello, J., Chen, A., Hawthorne, K., Marchionini, G., and Shaw, L. (2012, 10-14 June). The ResultsSpace collaborative search environment. Paper presented at the 12th ACM/IEEE-CS Joint Conference on Digital Libraries, Washington, DC.

[20] Sundaravej, T., Mirchandani, D., and Lederer, A. (2015) Synchronous collaboration technology use in teamwork. Paper presented at the 48th Hawaii International Conference on System Sciences (HICSS), 216-225.

[21] Malhotra, A., and Majchrzak, A. (2012). How virtual teams use their virtual workspace to coordinate knowledge. ACM Transactions on Management Information Systems, Vol. 3 No. 1.

[22] Kim, H., Suh, K. S., and Lee, U. K. (2013). Effects of collaborative online shopping on shopping experience through social and relational perspectives. Information \& Management, 50(4), 169-180.

[23] Razmerita, L. and Kirchner, K. (2015) Collaboration and E-Collaboration: A study of factors that influence perceived students' group performance. Paper presented at the 48th Hawaii International Conference on System Sciences (HICSS), 33-42.

[24] Borlund, P. (2016) A study of the use of simulated work task situations in interactive information retrieval evaluations: A meta-evaluation. Journal of Documentation, 72(3), 394-413.

[25] Byström, K., and Hansen, P. (2005). Conceptual framework for tasks in information studies. Journal of the American Society for Information Science and Technology, 56(10), 1050-1061.

[26] Joho, H., Jatowt, A., and Blanco, R. (2015). Temporal information searching behaviour and strategies. Information Processing \& Management, 51(6), 834-850.

[27] Jansen, B. J. and Schuster, S. (2011) Bidding on the buying funnel for sponsored search and keyword advertising. Journal of Electronic Commerce Research, 12(1), article 1.

[28] Liu, Z. and Jansen, B. J. (2016) ASK: A taxonomy of accuracy, social, and knowledge information seeking posts in social question and answering. Journal of the Association for Information Science and Technology. doi:10.1002/asi.23655.

[29] Standing, C., Holzweber, M., and Mattsson, J. (2016) Exploring emotional expressions in e-word-of-mouth from online communities. Information Processing \& Management, (52)5, 721-732. 